\title{
ESTIMACIÓN DEL TAMAÑO MUESTRAL PARA ESTUDIOS DE VARIABILIDAD MORFOLÓGICA IN SITU DE LIPPIA INTEGRIFOLIA (VERBENACEAE) EN EL PARQUE NACIONAL TALAMPAYA, LA RIOJA (ARGENTINA)
}

\author{
PAULA BRUNETTI1,5, CECILIA BRUNO ${ }^{2,5}$, RICARDO ZAPATA ${ }^{3}$, LORENA TORRES ${ }^{1}$, GLORIA BARBOZA ${ }^{4,5}$ Y \\ MARTA OJEDA ${ }^{1}$
}

\begin{abstract}
Summary: Brunetti P. C., C. Bruno, R. Zapata, L. Torres, G. Barboza \& M. Ojeda. 2014. Sample size estimation for in situ morphological variability studies of Lippia integrifolia (Verbenaceae) in Parque Nacional Talampaya, La Rioja (Argentina). Bonplandia 23(1): 15-23.
\end{abstract}

Lippia integrifolia "incayuyo" is an aromatic and medicinal shrub of economic importance, distributed from Bolivia to northwestern and central Argentina. It has shown a wide variation between individuals of the same species, which could be the result of interaction with the environment and / or be genetically determined. On the basis of this variability, characterization studies are needed for further agronomic evaluations, plant breeding, selection, conservation and reproduction of specimen with desirable characteristics. The aim of this study is to determine, using two estimation methods, the minimum number of individuals to be assessed that represents in situ morphological variability of a population of L. integrifolia. From a preliminary sampling conducted in the Parque Nacional Talampaya, in which 11 morphometric characters were registered in $L$. integrifolia, a parametric and a nonparametric estimation of the Minimum Sample Size was performed. Based on the morphometric characters evaluated here it is recommended a minimum sample size in between 35-40 individuals, determined by the characters that showed greater variability, with which the morphological variability of the species would be guaranteed.

Key words: Lippia integrifolia, in situ characterization, intraspecific variability, Minimum Sample Size.

Resumen: Brunetti P. C., C. Bruno, R. Zapata, L. Torres, G. Barboza \& M. Ojeda. 2014. Estimación del tamaño muestral para estudios de variabilidad morfológica in situ de Lippia integrifolia (Verbenaceae) en el Parque Nacional Talampaya, La Rioja (Argentina). Bonplandia 23(1): 15-23.

Lippia integrifolia "incayuyo" es un arbusto aromático y medicinal de interés económico cuya distribución abarca desde Bolivia hasta el noroeste y centro de Argentina. Presenta una amplia variabilidad intraespecífica, la cual podría ser el resultado de la interacción con el ambiente y/o estar determinada genéticamente. Debido a esta variabilidad, estudios de caracterización son necesarios para posteriores evaluaciones agronómicas, mejoramiento genético, selección,

${ }^{1}$ Fac. de Ciencias Agropecuarias, Universidad Nacional de Córdoba, Argentina. Cátedra de Genética. brunetti.paula@, gmail.com

${ }^{2}$ Fac. de Ciencias Agropecuarias, Universidad Nacional de Córdoba, Argentina. Cátedra de Estadística y Biometría.

${ }^{3}$ Fac. de Ciencias Agropecuarias, Universidad Nacional de Córdoba, Argentina. Cátedra de Manejo de Agrosistemas Marginales.

${ }^{4}$ Fac. de Ciencias Químicas, Universidad Nacional de Córdoba, Argentina. Cátedra de Botánica-IMBIV.

${ }^{5}$ CONICET 
reproducción y conservación de ejemplares con características deseables. El objetivo de este trabajo es determinar, utilizando dos métodos de estimación, el número de individuos mínimo a relevar in situ que representen la variabilidad morfológica de una población de $L$. integrifolia. A partir de un muestreo preliminar realizado en el Parque Nacional Talampaya en el que se registraron 11 caracteres morfométricos en $L$. integrifolia, se realizó una estimación paramétrica y una no paramétrica del Tamaño Muestral Mínimo. Sobre la base de los caracteres morfométricos aquí evaluados se recomienda un tamaño de muestra mínimo de entre 35 a 40 ejemplares, determinado por los caracteres que mostraron mayor variabilidad, con el cual se garantizaría poder captar la variabilidad morfológica de la especie.

Palabras clave: Lippia integrifolia, caracterización in situ, variabilidad intraespecífica, Tamaño Muestral Mínimo.

\section{Introducción}

Lippia integrifolia (Griseb.) Hieron. es un arbusto aromático, subleñoso, conocido popularmente como "incayuyo", "poleo", "pulco", "manzanillo", "té del inca", entre otros nombres; que forma parte de la vegetación nativa desde Bolivia hasta el noroeste y centro de Argentina (Zuloaga et al., 2008; Múlgura et al., 2012). La decocción de las hojas y flores son tradicionalmente usadas contra la dispepsia, indigestiones y dolores de estómago, como gastrálgico, diurético, emenagogo, antibiótico para infecciones de gonorrea, febrífuga, para el tratamiento de la tos y como sedativa (Toursarkissian, 1980; Pochettino et Martinez, 1998; Rondina et al., 2003; Barboza et al., 2009). Se encuentra dentro de las especies aromáticas nativas de mayor importancia económica, por su participación en yerbas compuestas y aperitivos, entre otros destinos. La demanda comercial y la no existencia de cultivos genera un alta presión de extracción sobre las poblaciones naturales en la Región Central y Noroeste de Argentina (INTA, 2007).

Desde el punto de vista taxonómico se ha evidenciado para la especie variación en su morfología -variabilidad intraespecífica-, por lo que Denham et al. (2006) han propuesto que lo que antes se consideraban dos especies, L. integrifolia y L. boliviana Rusby, sería sólo una, por encontrarse ejemplares con caracteres morfométricos intermedios. Dicha variabilidad podría ser atribuida a la interacción con el ambiente y/o estar determinada genéticamente (Mariotti, 1994; Cubero, 2003). Dado el valor económico potencial que posee esta especie es importante lograr una buena caracterización morfológica y conocer su variabilidad. El conocimiento de la variabilidad intraespecífica de una especie es utilizado para evaluaciones agronómicas en programas de mejoramiento genético vegetal, para selección y reproducción de materiales con características deseables, con el fin de fomentar y diversificar la producción agrícola (Allard, 1980; Utera \& Martínez, 1994; Jaramillo \& Baena, 2000; Cubero, 2003).

Para lograr una caracterización de la especie con un nivel de precisión óptimo, se debe establecer el número de individuos a relevar in situ. Un estudio previo para determinar el tamaño muestral debe llevarse a cabo antes de realizar mediciones de caracteres agronómicos con la finalidad de realizar pruebas estadísticas comparativas. Con este tipo de estudios preliminares se busca evitar una recolección de datos insuficiente, es decir, poco representativa de la población, tal que los estadísticos muestrales estimados sean sesgados, inconsistentes e ineficientes (Balzarini et al., 2012). Además, en estudios comparativos, si el tamaño de la muestra es menor que el necesario, se incrementa la probabilidad de cometer error de Tipo II $(\beta)$ y por lo tanto tener poca potencia en una prueba estadística inferencial. Por el contrario, si el tamaño muestral es mayor del requerido, se incurriría en el consumo innecesario de recursos económicos y/o de tiempo (Lapitan et al., 1979; Ambrosio et al., 2004; Confalonieri et al., 2009). Para estimar un tamaño muestral óptimo, se requiere conocer la variabilidad de los atributos o caracteres morfométricos de la especie. Para conocer 
la varianza de los parámetros morfométricos y reducir la incertidumbre de la estimación de los mismos, se realizó un muestreo preliminar de L. integrifolia en el Parque Nacional Talampaya (La Rioja, Argentina). El objetivo de este trabajo fue determinar, utilizando dos métodos de estimación, el número de individuos mínimo a relevar que representen la variabilidad fenotípica de una población de L. integrifolia, para futuros estudios de caracterización morfológica de la especie.

\section{Materiales y Métodos}

Datos experimentales. El sitio de muestreo fue el Parque Nacional Talampaya, ubicado en el centro-oeste de la Provincia de La Rioja, Argentina. Es un macizo rocoso de areniscas, con pronunciadas diferencias de temperatura, lluvias escasas pero torrenciales durante el verano (promedio de precipitación anual entre 80 a $100 \mathrm{~mm}$ ) y radiación solar intensa. Se encuentra vegetación principalmente arbustiva propia de zonas desérticas. Las mediciones se realizaron dentro del Parque, en las coordenadas $29^{\circ} 40^{\prime} 53^{\prime \prime}$ de latitud sur y $67^{\circ} 44^{\prime} 05^{\prime}$ ' de longitud oeste, altitud $1725 \mathrm{~m}$. El muestreo se realizó los días 16 y 17 de abril del año 2009, momento en el que las plantas de $L$. integrifolia se encontraban finalizando su ciclo de crecimiento, tanto vegetativo como reproductivo, y por lo tanto completamente desarrolladas. Se seleccionaron individuos al azar, distanciados entre ellos por más de $30 \mathrm{~m}$ para asegurar la individualidad de cada planta. Cada ejemplar fue considerado como una Unidad Taxonómica Operacional (UTO).

Los caracteres morfométricos evaluados in situ fueron: altura de la mata, diámetro mayor y menor de la canopia. Por otro lado se herborizó una rama de cada individuo relevado para medir en laboratorio sobre las hojas del $5^{\text {to }}$ y $10^{\mathrm{mo}}$ nudo, área foliar, largo y ancho de las hojas y la relación largo/ancho.

En este estudio preliminar se caracterizaron morfométricamente 40 UTOs, tomando como referencia estudios de caracterización fenotípica in situ en otras especies nativas argentinas (Ojeda, 2004; Chaves, 2012). A partir del $n=40$ UTOs se estimaron los estadísticos muestrales que fueron utilizados como valores iniciales en el cálculo del tamaño muestral.

Se depositó un ejemplar testigo en el herbario de la Fac. de Cs. Agropecuarias, Universidad Nacional de Córdoba (Argentina), bajo el registro ACOR CS 1187-1.

Métodos de estimación del Tamaño Muestral Mínimo (TMM). Se realizó una estimación paramétrica del TMM basado en la distribución t de Student según la siguiente fórmula:

$$
n \leq \frac{\left(2 . t_{1-\alpha / 2} \cdot S\right)^{2}}{c}
$$

donde, $n$ representa el mínimo tamaño muestral a estimar basado en la varianza muestral $\left(S^{2}\right)$, la confianza $(1-\alpha)$ determinada bajo el estadístico t de Student y la amplitud del intervalo de confianza (c). Sobre los 11 caracteres relevados se estimaron la media muestral y la varianza con $\operatorname{los} n=40$ UTOs. A partir de esta muestra se conformaron diferentes escenarios constituidos por la combinación de la amplitud del Intervalo de Confianza (IC) y diferentes niveles de precisión cambiando la confianza (1- $\alpha)$. Se estimaron los límites del IC (límite superior y límite inferior) para una distribución t de Student con 4 valores de confianza 0,$85 ; 0,90 ; 0,95$ y 0,99 .

También se realizó una estimación no paramétrica del TMM a partir de la muestra original de 40 ejemplares de L. integrifolia, para lo cual se aplicó el método de muestreo y re-muestreo con reposición bootstrap (Efron, 1979) para 8 tamaños muestrales diferentes. Se realizaron 4000 muestras bootstrap, variando el tamaño muestral (n) de 5 en 5 , desde un $n=5$ hasta $n=40$ individuos, con 500 muestras bootstrap por cada tamaño muestral. Este método no tiene en cuenta la distribución de los datos en el cálculo del tamaño muestral, como si se consideró al basarnos en la distribución $t$ de Student. La ventaja de poder contar con un método no paramétrico es la posibilidad de estimar un tamaño muestral independientemente de la distribución de las variables.

Se aplicó el test estadístico Shapiro-Wilks 
modificado (Mahibbur \& Govindarajulu, 1997) para comprobar el supuesto de normalidad en la distribución de los datos originales y el test de bondad de ajuste de Kolmogorov (Chakravart et al., 1967). El test de ShapiroWilks es considerado más potente que el test de Kolmogorov para probar la normalidad de las variables, sin embargo este último es más sensible a valores cercanos a la mediana que a los valores extremos de la distribución, además es independiente de la distribución postulada bajo hipótesis nula.

El software Infostat (Di Rienzo et al., 2013) fue utilizado para todos los procedimientos estadísticos realizados, el mismo cuenta con las herramientas necesarias y es de fácil aplicación para los objetivos de este trabajo.

\section{Resultados y Discusión}

\section{Análisis exploratorio}

Las variables asociadas al tamaño de las hojas -largo, ancho y área- mostraron desviación de la normalidad, tanto para las hojas pertenecientes al $5^{\text {to }}$ nudo, como también las del $10^{\text {mo }}$ nudo (Tabla 1 ), mientras que el resto de los caracteres presentaron distribución normal con el test de Shapiro-Wilks modificado. Este alejamiento de la distribución normal, asociado a la quinta y décima lámina, se corresponde con la alta variabilidad intraespecífica asociada a este órgano. Todos los caracteres también fueron sometidos al test de bondad de ajuste de Kolmogorov, el cual corroboró el ajuste

Tabla 1. Estadísticos asociados a los caracteres morfométricos: tamaño muestral (n), media, coeficiente de variación (CV), mínimo y máximo. Prueba de normalidad con el test de Shapiro-Wilks modificado y prueba de bondad de ajuste a una distribución normal con el Test de Kolmogorov.

\begin{tabular}{|c|c|c|c|c|c|c|c|c|c|c|c|}
\hline \multirow[b]{2}{*}{ Variable } & \multirow[b]{2}{*}{ Unidad } & \multirow[b]{2}{*}{$\mathbf{n}$} & \multirow[b]{2}{*}{ Media } & \multirow[b]{2}{*}{$\mathbf{C V}$} & \multirow[b]{2}{*}{ Mín } & \multirow[b]{2}{*}{ Máx } & \multicolumn{2}{|c|}{$\begin{array}{c}\text { Test de } \\
\text { Normalidad } \\
\end{array}$} & \multicolumn{3}{|c|}{ Test de bondad de ajuste } \\
\hline & & & & & & & W & $\begin{array}{c}\mathbf{p} \\
\text { (Unilateral } \\
\text { D)* }\end{array}$ & Ajuste & $\begin{array}{c}\text { Estadístico } \\
\text { D }\end{array}$ & p-valor $\dagger$ \\
\hline $\begin{array}{l}\text { Altura } \\
\text { mata }\end{array}$ & $\mathrm{m}$ & 40 & 0,92 & 29,86 & 0,40 & 1,50 & 0,95 & & Normal $(0,92 ; 0,08)$ & 0,11 & \\
\hline $\begin{array}{l}\text { Diámetro } \\
\text { Mayor }\end{array}$ & $\mathrm{m}$ & 40 & 0,82 & 35,88 & 0,12 & 1,40 & 0,94 & & Normal $(0,82 ; 0,09)$ & 0,14 & \\
\hline & $\mathrm{m}$ & 40 & 0,62 & 38,68 & 0,20 & 1,20 & 0,94 & & Normal $(0,62 ; 0,06)$ & 0,13 & \\
\hline & $\mathrm{cm}$ & 37 & 1,55 & 47,81 & 0,60 & 5,11 & 0,75 & $\mathrm{~S}(\mathrm{p}<0,05)$ & Normal $(1,55 ; 0,55)$ & 0,18 & \\
\hline $\begin{array}{l}\text { Ancho } 5^{\text {ta }} \\
\text { hoja } \\
\text { Largo/ }\end{array}$ & $\mathrm{cm}$ & 37 & 0,39 & 33,91 & 0,17 & 0,95 & 0,86 & $\mathrm{~S}(\mathrm{p}<0,05)$ & Normal $(0,39 ; 0,02)$ & 0,15 & \\
\hline $\begin{array}{l}\text { Ancho } 5^{\text {ta }} \\
\text { hoja }\end{array}$ & & 37 & 3,99 & 24,62 & 2,21 & 6,16 & 0,93 & & Normal $(3,99 ; 0,96)$ & 0,13 & \\
\hline $\begin{array}{l}\text { Área } 5^{\text {ta }} \\
\text { hoja }\end{array}$ & $\mathrm{cm}^{2}$ & 37 & 0,47 & 105,57 & 0,11 & 3,19 & 0,55 & $\mathrm{~S}(\mathrm{p}<0,05)$ & Normal $(0,47 ; 0,25)$ & 0,27 & $\mathrm{~K}(\mathrm{p}<0,05)$ \\
\hline $\begin{array}{l}\text { Largo } 10^{\text {ma }} \\
\text { hoja }\end{array}$ & $\mathrm{cm}$ & 37 & 2,58 & 42,79 & 1,08 & 6,44 & 0,90 & $\mathrm{~S}(\mathrm{p}<0,05)$ & Normal $(2,58 ; 1,22)$ & 0,18 & \\
\hline $\begin{array}{l}\text { Ancho } \\
10^{\mathrm{ma}} \text { hoja } \\
\text { Largo/ }\end{array}$ & $\mathrm{cm}$ & 37 & 0,56 & 36,62 & 0,33 & 1,29 & 0,88 & $\mathrm{~S}(\mathrm{p}<0,05)$ & Normal $(0,56 ; 0,04)$ & 0,14 & \\
\hline Ancho & & 37 & 4,61 & 23,82 & 2,50 & 7,24 & 0,96 & & Normal $(4,61 ; 1,21)$ & 0,09 & \\
\hline $\begin{array}{l}10^{\mathrm{ma}} \text { hoja } \\
\text { Área } 10^{\mathrm{ma}} \\
\text { hoja }\end{array}$ & $\mathrm{cm}^{2}$ & 37 & 1,12 & 87,55 & 0,27 & 5,73 & 0,72 & $\mathrm{~S}(\mathrm{p}<0,05)$ & Normal $(1,12 ; 0,97)$ & 0,19 & \\
\hline
\end{tabular}

* S indica la no normalidad acorde al test de Shapiro-Wilks modificado por Mahibbur y Govindarajulu (1997).

$\dagger \mathrm{K}$ indica la falta de ajuste a la distribución normal según Kolmogorov. 
a una distribución normal en todos los casos ( $p>0,05)$, excepto para el carácter área de la $5^{\text {ta }}$ hoja. Si bien, éste último es menos potente que el primero, al ser más sensible a valores cercanos a la media y estar menos afectado que el test de Shapiro-Wilks a valores extremos, sólo detectó falta de normalidad en ese carácter.

Los valores promedios y su variabilidad, expresada como Coeficiente de Variación (CV) se observan en la Tabla 1, para los 11 caracteres morfológicos evaluados in situ sobre los 40 UTOs. El área foliar de la $5^{\text {ta }}$ y $10^{\text {ma }}$ hoja mostraron la mayor variabilidad -medida como desvío estándar- con respecto a los valores promedio, con un CV de $105 \%$ y $87 \%$, respectivamente. Esto se corresponde con lo informado por otros autores (Denham et al., 2006) quienes encontraron que los caracteres ancho de la lámina del $5^{\text {to }}$ y $10^{\text {mo }}$ nudo y largo de la lámina del $5^{\text {to }}$ nudo -entre otros caracteres- estuvieron dentro de las variables que más inercia aportaron al componente uno, en el Análisis de Componentes Principales, al evaluar 40 UTOs herborizados de L. integrifolia de distinto origen geográfico.

\section{Determinación del mínimo tamaño muestral}

La variable Ancho $5^{\text {ta }}$ hoja presentó los TMM más grandes respecto al resto de los caracteres, en la mayoría de los escenarios evaluados (Tabla 2). Para una probabilidad del $85 \%$, que resulta en el IC más acotado, y el máximo nivel de significancia de 0,01 se obtuvieron los TMM de mayor magnitud, siendo la variable Ancho $5^{\text {ta }}$ hoja la que presentó el mayor TMM de 147 individuos, seguida por el Diámetro Mayor de la canopia con 141 muestras.

En la práctica, habitualmente se utilizan valores de probabilidad de IC del 95\%, y un nivel de significancia de la estimación de $0,05(1-\alpha)$. Comparando en este nivel los 11 caracteres morfométricos evaluados in situ, los TMM requeridos se encuentran en el rango de 30 a 40 individuos, registrándose para la variable Diámetro Menor de la canopia el tamaño muestral más alto de 41 muestras (Fig. 1).

Para cada muestra bootstrap obtenida por remuestreo se estimó el $\mathrm{CV}$, para cada carácter

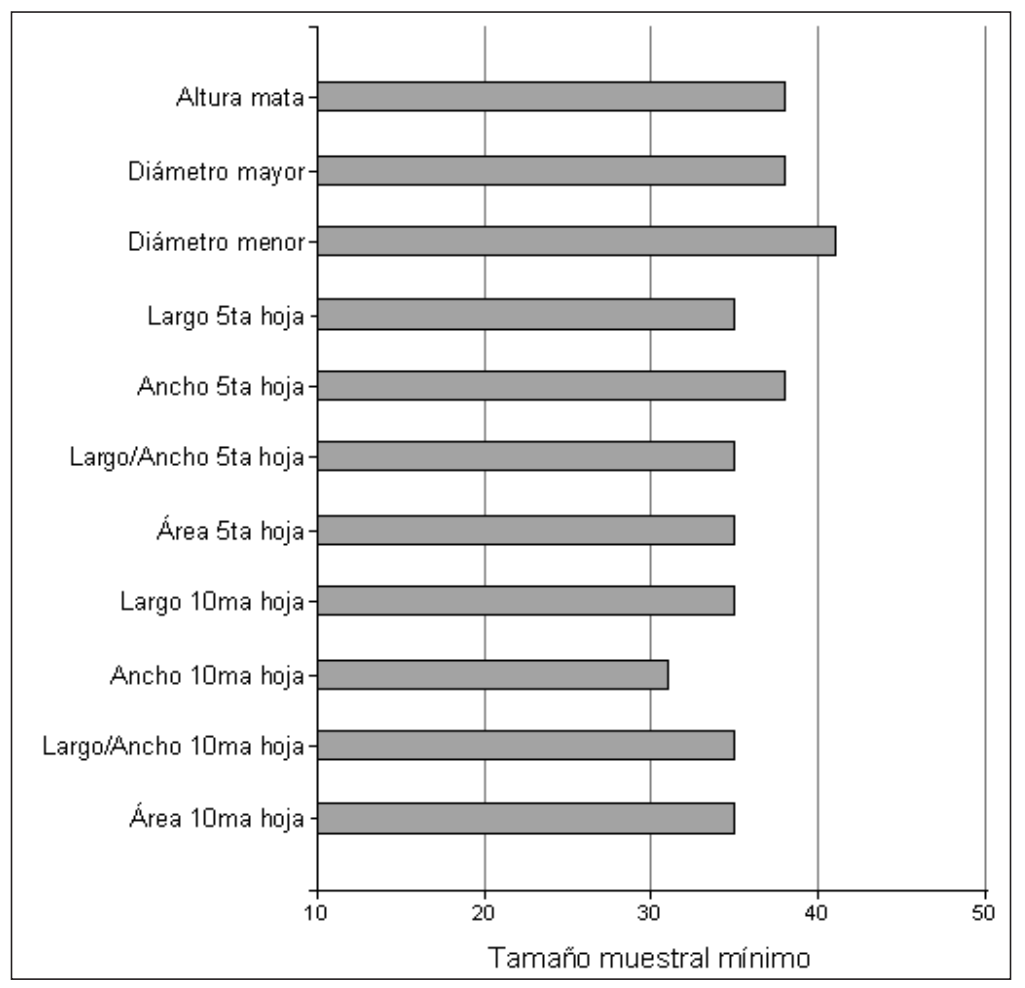

Fig. 1. Tamaños muestrales obtenidos para 11 caracteres morfométricos de Lippia integrifolia, mediante estimación paramétrica para una Confianza del Intervalo del 95\% y con un nivel de significancia $(\alpha)$ de 0,05 . 
Tabla 2. Estimación paramétrica del tamaño muestral basado en la distribución t de Student, calculados para cuatro Intervalos de Confianza (IC) y tres niveles de precisión.

\begin{tabular}{|c|c|c|c|c|c|}
\hline \multirow[t]{2}{*}{ Carácter morfométrico } & \multirow[t]{2}{*}{$\begin{array}{l}\text { Confianza del } \\
\text { Intervalo (\%) }\end{array}$} & \multirow[t]{2}{*}{$\begin{array}{c}\text { Amplitud IC } \\
\text { estimado }\end{array}$} & \multicolumn{3}{|c|}{$\begin{array}{l}\text { Mínimo tamaño muestral (n) estimado } \\
\text { para diferentes niveles de significancia } \\
(\alpha)\end{array}$} \\
\hline & & & $\mathbf{0 , 0 1}$ & 0,05 & $\mathbf{0 , 1}$ \\
\hline \multirow{4}{*}{ Altura } & 99 & 0,24 & 37 & 21 & 15 \\
\hline & 95 & 0,18 & 66 & 38 & 27 \\
\hline & 90 & 0,15 & 94 & 55 & 38 \\
\hline & 85 & 0,13 & 126 & 73 & 51 \\
\hline \multirow{4}{*}{ Diámetro Mayor } & 99 & 0,25 & 38 & 22 & 16 \\
\hline & 95 & 0,19 & 66 & 38 & 27 \\
\hline & 90 & 0,15 & 106 & 61 & 43 \\
\hline & 85 & 0,13 & 141 & 82 & 58 \\
\hline \multirow{4}{*}{ Diámetro Menor } & 99 & 0,21 & 36 & 24 & 15 \\
\hline & 95 & 0,15 & 71 & 41 & 29 \\
\hline & 90 & 0,13 & 94 & 55 & 38 \\
\hline & 85 & 0,11 & 132 & 76 & 54 \\
\hline \multirow{4}{*}{ Largo $5^{\text {ta }}$ hoja } & 99 & 0,66 & 34 & 19 & 14 \\
\hline & 95 & 0,49 & 61 & 35 & 25 \\
\hline & 90 & 0,41 & 87 & 50 & 35 \\
\hline & 85 & 0,36 & 113 & 65 & 46 \\
\hline \multirow{4}{*}{ Ancho $5^{\text {ta }}$ hoja } & 99 & 0,12 & 37 & 21 & 15 \\
\hline & 95 & 0,09 & 66 & 38 & 27 \\
\hline & 90 & 0,07 & 108 & 63 & 44 \\
\hline & 85 & 0,06 & 147 & 85 & 60 \\
\hline \multirow{4}{*}{ Largo/Ancho $5^{\text {ta }}$ hoja } & 99 & 0,87 & 34 & 19 & 14 \\
\hline & 95 & 0,65 & 60 & 35 & 25 \\
\hline & 90 & 0,54 & 87 & 51 & 36 \\
\hline & 85 & 0,47 & 115 & 67 & 47 \\
\hline \multirow{4}{*}{ Área $5^{\text {ta }}$ hoja } & 99 & 0,44 & 34 & 20 & 14 \\
\hline & 95 & 0,33 & 61 & 35 & 25 \\
\hline & 90 & 0,28 & 68 & 40 & 28 \\
\hline & 85 & 0,24 & 115 & 67 & 47 \\
\hline \multirow{4}{*}{ Largo $10^{\text {ma }}$ hoja } & 99 & 0,98 & 34 & 20 & 14 \\
\hline & 95 & 0,73 & 61 & 35 & 25 \\
\hline & 90 & 0,61 & 87 & 50 & 35 \\
\hline & 85 & 0,53 & 115 & 67 & 47 \\
\hline \multirow{4}{*}{ Ancho $1^{\mathrm{ma}}$ hoja } & 99 & 0,19 & 29 & 17 & 12 \\
\hline & 95 & 0,14 & 54 & 31 & 22 \\
\hline & 90 & 0,12 & 74 & 43 & 30 \\
\hline & 85 & 0,10 & 106 & 61 & 43 \\
\hline \multirow{4}{*}{ Largo/Ancho $10^{\mathrm{ma}}$ hoja } & 99 & 0,98 & 33 & 19 & 14 \\
\hline & 95 & 0,73 & 60 & 35 & 25 \\
\hline & 90 & 0,61 & 86 & 50 & 35 \\
\hline & 85 & 0,53 & 114 & 66 & 47 \\
\hline \multirow{4}{*}{ Área $10^{\mathrm{ma}}$ hoja } & 99 & 0,87 & 34 & 20 & 14 \\
\hline & 95 & 0,65 & 61 & 35 & 25 \\
\hline & 90 & 0,54 & 88 & 51 & 36 \\
\hline & 85 & 0,47 & 117 & 67 & 48 \\
\hline
\end{tabular}




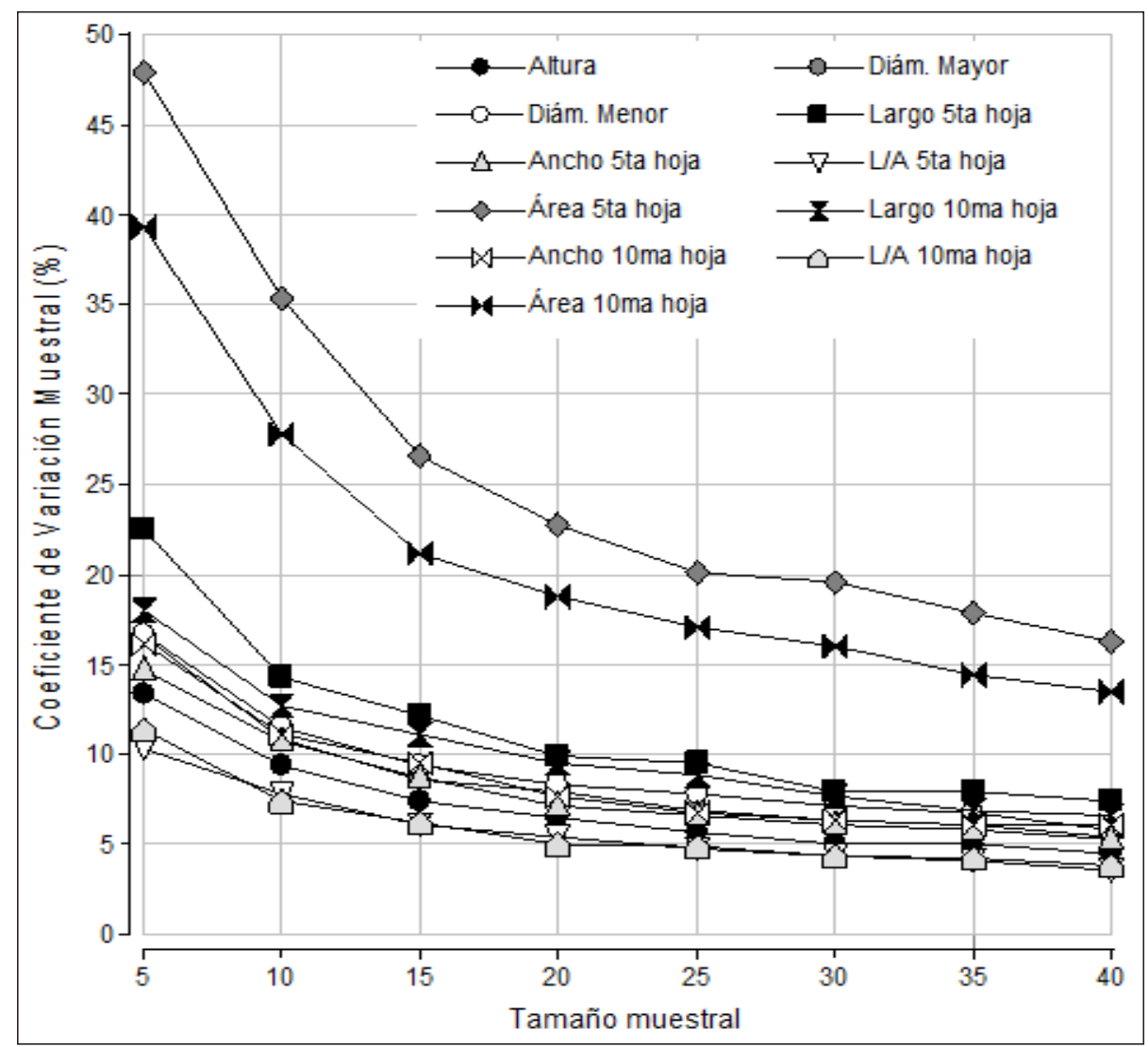

Fig. 2. Método no paramétrico de muestreo y re-muestreo con reposición bootstrap, para 8 tamaños muestrales, aplicado a la muestra original de Lippia integrifolia.

morfométrico. En la Fig. 2 se observa la disminución del $\mathrm{CV}$ a medida que aumenta el tamaño de la muestra. Un mayor valor de este estimador de la varianza está relacionado a las variables área de la $5^{\text {ta }}$ y $10^{\text {ma }}$ hoja, para las cuales un CV del $20 \%$ se alcanza con un tamaño de muestra de entre 30 a 35 ejemplares.

Existen trabajos en donde se ha calculado el tamaño muestral en especies cultivadas (Lapitan et al., 1979; Confalonieri, 2004; Confalonieri et al., 2009); pero no sucede lo mismo para especies silvestres, en donde el número de individuos a relevar para trabajos de caracterización in situ se ha establecido arbitrariamente y los tamaños de muestra usados son variados (Ojeda, 2004; Wang et Yu, 2007; Bayuelo-Jiménez et al., 2006; Janyszek et al., 2008; Cserháti et al., 2012; Chaves, 2012). Los tamaños de muestra obtenidos para L. integrifolia en la Fig. 1, son en general mayores que aquellos encontrados para especies cultivadas, lo cual se podría atribuir al hecho de que L. integrifolia es una especie silvestre cuya variabilidad, debida a la genética o a la plasticidad, es mayor que en especies cultivadas ya mejoradas y por consiguiente con una variabilidad morfológica esperada y observada menor.

\section{Conclusión}

Para estudios de caracterización in situ de L. integrifolia -considerando los caracteres morfométricos aquí evaluados- se recomienda un tamaño de muestra mínimo de entre 35 a 40 UTOs, determinado por los caracteres que mostraron mayor variabilidad, con el cual se garantizaría poder captar la variabilidad morfológica de los individuos.

Este trabajo sienta las bases para futuras 
determinaciones del número de individuos a relevar para estudios de caracterización in situ de poblaciones silvestres y para selección de caracteres diferenciales en programas de mejoramiento.

\section{Bibliografía}

ALLARD, R. 1980. Principios de la Mejora Genética de las Plantas. Ed. Omega. Barcelona, España. 498 pp.

AMBROSIO, L., L. IGLESIAS, C. MARIN \& J. P. DEL MONTE. 2004. Evaluation of sampling methods and assesment of the simple size to estimate the weed seedbank in soil, taking into account spatial variability. Weed Res. 44: 224-236.

BALZARINI, M., J. DI RIENZO, M. TABLADA, L. GONZALEZ, C. BRUNO, M. CÓRDOBA, W. ROBLEDO \& F. CASANOVES. 2012. Estadística y Biometría. Ilustraciones del uso de Infostat en problemas de agronomía. Universidad Nacional de Córdoba. 400 pp.

BARBOZA, G., J. CANTERO, C. NÚÑEZ, A. PACCIARONI \& L. ARIZA ESPINAR. 2009. Medicinal Plants: A general review and a phytochemical and ethnopharmacological screening of the native Argentine flora. Kurtziana 34: 7-365.

BAYUELO-JIMÉNEZ, J., J. LOZANO RICO \& I. OCHOA. 2006. Caracterización morfológica de Byrsonima crassifolia (L.) Kunth nativa de Churumuco, Michoacán, México. Rev. Fitotec. Mex. 29: 31-36.

CHAKRAVARTI, I. M., LAHA R. C. \& J. ROY. 1967. Handbook of Methods of Applied Statistics I: 392394. John Wiley and Sons, New York.

CHAVES, A. G. 2012. Variabilidad morfológica de poblaciones de Baccharis spp., "carquejas" (Asteraceae) de las Sierras de Córdoba y San Luis (Argentina). Tesis de maestría, Facultad de Ciencias Agropecuarias, Universidad Nacional de Córdoba. 59 pp.

CONFALONIERI, R. 2004. A Jackknife-derived visual approach for sample size determination. Riv. Ital. Agromet. 1: 9-13.

- A. PEREGO, M. E.CHIODINI, B. SCAGLIA, A. S. ROSENMUND \& M. ACUTIS. 2009. Analysis of sample size for variables related to plant, soil, and soil microbial respiration in a paddy rice field. Field Crop. Res. 113: 125-130.

CSERHÁTI, B., K. JUHOS, A. BEGYIK, P. RADÁCSI, É. NÉMETH \& K. SZABÓ. 2012. In situ morphological variability of wild marjoram (Origanum vulgare L.) populations in Hungary. Acta Alimentaria 41: 12-23.

CUBERO, J. 2003. Introducción a la Mejora Genética Vegetal. $2^{\circ}$ Edición. Ed. Mundi-Prensa. Madrid, España. 567 pp.
DENHAM, S., M. MÚlgURA DE ROMERO, A. SLANIS \& E. BULACIO. 2006. Lippia integrifolia versus L. boliviana (Verbenaceae). Darwiniana 44: 363-374.

DI RIENZO, J. A., F. CASANOVES, M. G. BALZARINI, L. GONZALEZ, M. TABLADA \& C. W. ROBLEDO. 2013. InfoStat versión 2013. Grupo InfoStat, Facultad de Ciencias Agropecuarias, Universidad Nacional de Córdoba, Argentina. URL http://www.infostat.com.ar

EFRON, B. 1979. Bootstrap methods: Another look at the jackknife. Ann. Stat. 7: 1-26.

INSTITUTO NACIONAL DE TECNOLOGÍA AGROPECUARIA (INTA). 2007. Parte de Prensa. Desarrollo de tecnologías innovativas para la exploración conservación, evaluación y utilización de plantas aromáticas nativas. Estación Experimental La Rioja, Argentina.

JANYSZEK, M., A. M. JAGODZINSKI, S. JANYSZEK \& D. WRONSKA-PILAREK. 2008. Morphological variability of Carex spicata Huds. utricles among plant communities. Flora 203: 386-395.

JARAMILLO, S. \& M. BAENA. 2000. Material de apoyo a la capacitación en conservación ex situ de recursos fitogenéticos. Instituto Internacional de Recursos Fitogenéticos, Cali, Colombia. 201 pp.

LAPITAN, R. L., J. M. DABU \& E. L. ROSARIO. 1979. Optimum sample size and best sampling design for maximum accuracy in measuring yield components of sugarcane. Philipp. J. Crop Sci. 4: 78-82.

MAHIBBUR, R. M. \& Z. GOVINDARAJULU. 1997. A modification of the test of Shapiro and Wilks for normality. J. Appl. Stat. 24: 219-235.

MARIOTTI, J. A. 1994. La interacción genotipo-ambiente, su significado e importancia en el mejoramiento genético y en la evaluación de cultivares. Serie Monográfica $\mathrm{N}^{\circ}$ 1. INTA-E.E.A. Famaillá. 39 pp.

MULGURA, M. E., N. O'LEARY \& A. ROTMAN. 2012. Verbenaceae. En F. Zuloaga, \& A. Anton (eds.) Flora Argentina 14: 1-220.

OJEDA, M. S. 2004. Caracterización de poblaciones y avances en la domesticación de peperina Minthostachys mollis (Kunth) Griseb. Tesis doctoral, Facultad de Ciencias Agropecuarias, Universidad Nacional de Córdoba. Argentina. 132 pp.

POCHETTINO, M. \& M. MARTINEZ. 1998. Aporte al conocimiento actual de las plantas medicinales en Argentina: estudio etnobotánico en el Dep. Molinos, provincia Salta, Argentina. En A. Amat (coord.) Farmacobotánica y Farmacognosia en Argentina 1980-1998: 55-86.

RONDINA, R., A. BANDONI \& J. COUSSIO. 2003. Plantas silvestres argentinas con reconocidas propiedades medicinales o tóxicas. OEA-Cyted: Buenos Aires, CD-ROM.

TOURSARKISSIAN, M.1980. Plantas medicinales de la Argentina, Pp. 63-178. Ed. H. Sur. Buenos Aires, Argentina. 
UTERA, L. \& Y. MARTINEZ. 1994. Caracterización in situ de zapote (Pouteria sapota Jacq.). Tikalia 12: 35-50.

WANG, J. \& D. YU. 2007. Influence of sediment fertility on morphological variability of Vallisneria spiralis L. Aquat. Bot. 87: 127-133.
ZULOAGA F., O. MORRONE, \& M. J. BELGRANO. 2008. Catálogo de las Plantas Vasculares del Cono Sur (Argentina, Sur de Brasil, Chile, Paraguay y Uruguay). Monogr. Syst. Bot. Missouri Bot. Gard. 107: 3126 .

Original recibido el 11 de octubre de 2013; aceptado el 30 de noviembre de 2013. 
